\title{
Níveis de Lisina Mantendo a Relação Aminoacídica para Frangos de Corte no Período de 1 a 21 Dias de Idade, em Diferentes Ambientes Térmicos ${ }^{1}$
}

\section{Paulo Segatto Cella ${ }^{2}$, Juarez Lopes Donzele ${ }^{3}$, Rita Flávia Miranda de Oliveira ${ }^{3}$, Luiz Fernando Teixeira Albino ${ }^{3}$, Aloízio Soares Ferreira ${ }^{3}$, Paulo Cezar Gomes ${ }^{3}$, Sandra Roselí Valerio ${ }^{4}$, Lourdes Romão Apolonio 5}

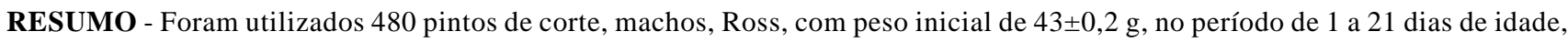
distribuídos em um delineamento experimental em esquema fatorial 2 x 4, dois ambientes (conforto térmico e alta temperatura) e quatro níveis de lisina total $(1,1 ; 1,2 ; 1,3 ; \mathrm{e} 1,4 \%)$, com seis repetições e 10 aves por unidade experimental. Verificou-se efeito do ambiente sobre o consumo de ração (CR) e o ganho de peso (GP), que foram maiores no ambiente de conforto, enquanto a conversão alimentar (CA) e a eficiência de utilização de lisina foram melhores no ambiente de alta temperatura. No ambiente de conforto térmico, o GP e o consumo de lisina aumentaram com os níveis de lisina da ração e a eficiência de utilização de lisina para GP melhorou de forma linear. Na alta temperatura, os níveis de lisina da ração influenciaram o GP e o consumo de lisina, que aumentaram de forma linear, e melhoraram também, de forma linear, a eficiência de utilização de lisina para GP. A conversão alimentar melhorou de forma quadrática até o nível de 1,29\% de lisina. Apesar do aumento linear, o modelo LRP foi o que melhor se ajustou aos dados de GP, estimando em 1,24\% o nível de lisina, a partir do qual ocorreu um platô. A alta temperatura ambiente influenciou negativamente o GP e CR, embora tenha melhorado a CA. Frangos de corte de 1 a 21 dias exigiram 1,4 e 1,29\% de lisina total, respectivamente, nos ambientes de conforto e alta temperatura.
\end{abstract}

Palavras-chave: conforto, desempenho, estresse por calor, pintos de corte

\section{Levels of Lysine Maintaining the Amino Acid Relation for Broilers from 1 to 21 Days of Age in Different Thermal Environments}

\begin{abstract}
Four hundred and eighty Ross broiler male, with initial weight of $43 \pm 0.2 \mathrm{~g}$, from 1 to 21 days of age, were allotted to an experimental design in $2 \times 4$ factorial arrangement, two environments (high temperature and thermal comfort) and four levels of total lysine (1.1, 1.2, 1.3 and 1.4\%) with six replicates and ten birds/experimental unity. Feed intake (FI) and weight gain (WG) were higher in the thermoneutral environment, while the feed: gain ratio (F/G) and the efficiency of utilization of lysine were better at the high environmental temperature. At the thermal comfort environment, the WG and lysine intake increased with the dietary lysine levels in the diet and the efficiency of lysine utilization for WG linearly improved. At the high temperature environment, the lysine levels influenced the WG and lysine intake, which linearly increased, and also improved, in a linear response, the efficiency of lysine utilization for WG. The feed: gain ratio improved in a quadratic way up to the level of $1.29 \%$ of lysine. Despite of the linear response, the LRP model was the one that better fitted the WG data, estimating the lysine level in $1.24 \%$, the plateau point. The high temperature environment negatively influenced WG and FI, even so improved the FC. Broilers from 1 to 21 days of age need 1.4 and $1.29 \%$ of total lysine, in thermal comfort and high temperature environments, respectively.
\end{abstract}

Key Words: broiler chick, heat stress, performance, thermal comfort environment

\section{Introdução}

O progresso genético na taxa de crescimento, na conversão alimentar e no rendimento de carcaça em frangos de corte tem sido um desafio constante para os nutricionistas. Considerando-se que a expressão fenotípica do potencial genético depende do ambiente e da nutrição, torna-se importante conhecer as exi- gências nutricionais das aves em processo de melhoramento, para se obter máximo desempenho das mesmas (BARBOZA, 1998).

$\mathrm{O}$ ambiente térmico influencia a produtividade dos animais, por alterar a troca de calor com o ambiente, o consumo de alimentos, o ganho de peso corporal e a exigência de proteína da ração, entre outros. Assim, para qualquer alteração da temperatura

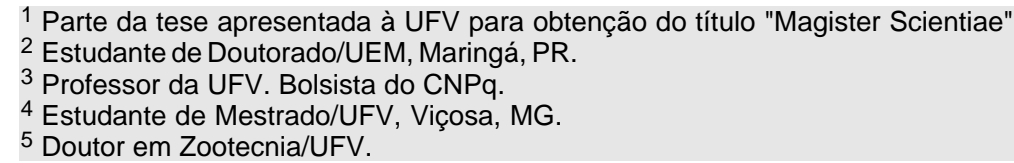


ambiente fora da faixa de conforto térmico, as aves podem necessitar de ajustes, sejam de natureza física, fisiológica ou comportamental, para se adaptarem à nova condição do meio (CURTIS, 1983). Dessa forma, o ambiente que compreende os fatores físicos, químicos e biológicos que envolvem o corpo do animal é considerado confortável, quando permitir a manutenção do equilíbrio térmico, sem comprometimento do rendimento animal.

A produção comercial de aminoácidos sintéticos viabilizou a redução dos teores de proteína bruta $(\mathrm{PB})$ das rações, devido à facilidade e disponibilidade das inclusões deles na ração, o que pode permitir redução nas perdas na forma de calor (incremento calórico). Segundo PENZ JR.(1989), níveis elevados de proteína na ração aumentam a carga de calor a ser dissipado, o que pode comprometer o desempenho dos animais em condições de alta temperatura. Assim, a redução no catabolismo da proteína resulta em decréscimo na produção de calor e ajuda a ave a manter seu balanço energético, em condições de elevadas temperaturas.

A suplementação de aminoácidos sintéticos às rações comerciais tem proporcionado facilidades no ajuste das formulações dessas rações, por possibilitar melhor balanço entre os aminoácidos essenciais. As exigências dos aminoácidos é estimada com base em um aminoácido referência. O aminoácido usado tem sido a lisina, cuja escolha é devida principalmente ao fato de que, em seu metabolismo, é usada quase que exclusivamente para acréscimo de proteína corporal (PACK, 1995).

Com base nessas informações, conduziu-se este trabalho com o objetivo de determinar as exigências de lisina, mantendo a relação aminoacídica, de frangos de corte de 1 a 21 dias mantidos em diferentes ambientes térmicos.

\section{Material e Métodos}

Foram utilizados 480 pintos de 1 dia de idade, machos, da linhagem Ross, com peso inicial médio de $43,0 \pm 0,2 \mathrm{~g}$, distribuídos em delineamento experimental inteiramente casualizado, em esquema fatorial $2 \times 4$, constituído de dois ambientes (conforto térmico e alta temperatura) e quatro níveis de lisina total $(1,1,1,2$; 1,3; e 1,4\%), com seis repetições e 10 aves por unidade experimental.

As rações experimentais, cujas composições estão apresentadas na Tabela 1, foram formuladas para atender as exigências nutricionais das aves em proteína, energia, minerais e vitaminas, segundo ROSTAGNO et al. (1996), exceto a lisina total $(1,165 \%$, segundo ROSTAGNO et al., 1996). As rações foram suplementadas com L-lisina $\mathrm{HCl} 78,4 \%$ $(0,16 ; 0,34 ; 0,52$; e 0,70$)$, resultando em rações com 1,10 e 0,$90 ; 1,20$ e 1,$09 ; 1,30$ e 1,20 ; e 1,40 e $1,29 \%$ de lisina total e digestível, respectivamente. Os aminoácidos DL-metionina, L-treonina, L-isoleucina, L-arginina e L-triptofano foram adicionados às rações em quantidades necessárias para atender ou exceder o padrão de proteína ideal, segundo a relação de BAKER e HAN (1994) para aminoácidos digestíveis, em que a lisina equiivale a $100 \%$; metionina, $36 \%$; metionina + cistina, $72 \%$; arginina, $105 \%$; treonina, $67 \%$; triptofano, $16 \%$; isoleucina, $67 \%$; e valina, $77 \%$. A composição em aminoácidos digestíveis foi calculada com base nos coeficientes de digestibilidade dos aminoácidos dos ingredientes contidos nas tabelas RHODIMET... (1993).

As aves receberam ração e água à vontade, durante todo o período experimental e foram alojadas em baterias metálicas, divididas em compartimentos de $0,72 \mathrm{~m}^{2}$, dotados de bebedouros e comedouros tipo calha, mantidas em câmaras climatizadas para conforto térmico e alta temperatura.

O monitoramento da temperatura e umidade relativa do ar de cada ambiente foi feito por meio de termômetro de bulbo seco e bulbo úmido e termômetro de globo negro, colocados à altura intermediária da bateria. As leituras dos termômetros foram realizadas diariamente, duas vezes ao dia $(8$ e $18 \mathrm{~h})$. Posteriormente, estes valores foram convertidos em valor único - Índice de Temperatura de Globo e Umidade (ITGU), segundo BUFFINGTON et al. (1977), para caracterização ambiental em conjunto, segundo a equação:

$$
\text { ITGU }=\text { Tgn }+0,36 \text { Tpo }-330,08
$$

em que Tgn é temperatura de globo negro $\left({ }^{\circ} \mathrm{K}\right)$ e Tpo, temperatura de ponto de orvalho $\left({ }^{\circ} \mathrm{K}\right)$.

O programa de luz adotado durante todo o período experimental foi o contínuo ( 24 horas de luz artificial), fazendo-se uso de duas lâmpadas fluorescentes de $75 \mathrm{~W}$, por câmara.

As aves foram pesadas no início e no final do período experimental para determinação do ganho de peso. O consumo de ração foi calculado considerando-se a ração fornecida, no período experimental, os desperdícios a as sobras das rações nos comedouros. A conversão alimentar foi estimada pela razão entre o consumo de ração e o ganho de peso das aves.

A eficiência de utilização de lisina para ganho foi 
Tabela 1 - Composição centesimal das rações experimentais para frangos corte de 1 a 21 dias Table 1 - Centesimal composition of the experimental diets for broilers from 1 to 21 days of age

\begin{tabular}{|c|c|c|c|c|}
\hline \multirow[b]{2}{*}{$\begin{array}{l}\text { Ingredientes }(\%) \\
\text { Ingredient }\end{array}$} & \multicolumn{4}{|c|}{$\begin{array}{c}\text { Níveis de lisina total }(\%) \\
\text { Total lysine level }\end{array}$} \\
\hline & 1,10 & 1,20 & 1,30 & 1,40 \\
\hline Milho (Corn grain) & 60,18 & 60,96 & 60,73 & 61,36 \\
\hline Farelo de soja (Soybean meal) & 27,00 & 25,00 & 24,00 & 22,00 \\
\hline Farelo de glúten de milho (Corn gluten meal) & 7,00 & 7,00 & 7,00 & 7,00 \\
\hline Fosfato bicálcico (Dicalcium phosphate) & 2,10 & 2,10 & 2,10 & 2,10 \\
\hline Calcário(Limestone) & 1,20 & 1,20 & 1,20 & 1,20 \\
\hline Caulim(Caulin) & 0,41 & 0,99 & 1,58 & 2,25 \\
\hline Bicarbonato de sódio (Sodiun bicarbonate) & 0,10 & 0,10 & 0,10 & 0,10 \\
\hline $\mathrm{Sal}$ (Salt) & 0,40 & 0,40 & 0,40 & 0,40 \\
\hline Óleo degomado (Soybean oil) & 0,70 & 0,70 & 0,70 & 0,70 \\
\hline Mistura vitamínica (Vitamin mix) ${ }^{1}$ & 0,50 & 0,50 & 0,50 & 0,50 \\
\hline Mistura mineral (Mineral mix) ${ }^{2}$ & 0,05 & 0,05 & 0,05 & 0,05 \\
\hline Aditivos(Additives) ${ }^{3}$ & 0,10 & 0,10 & 0,10 & 0,10 \\
\hline L - Lisina HCL (HCL L-lysine) & 0,16 & 0,34 & 0,52 & 0,70 \\
\hline L - Arginina (L-arginine) & 0,07 & 0,23 & 0,38 & 0,54 \\
\hline DL- Metionina ( $D L-$ methionine) & 0,03 & 0,13 & 0,21 & 0,31 \\
\hline L - Treonina (L-threonine) & - & 0,11 & 0,21 & 0,32 \\
\hline L - Isoleucina (L-isoleucine) & - & 0,09 & 0,22 & 0,35 \\
\hline L - Triptofano (L-triptofano) & - & - & - & 0,02 \\
\hline \multicolumn{5}{|l|}{ Composição calculada (Calculated composition) } \\
\hline Energia met. (kcal/kg) (Met. energy, $k c a l / k g)$ & 3000 & 3000 & 3000 & 3000 \\
\hline Proteína bruta (\%) (Crude protein, \%) & 21,9 & 21,7 & 21,8 & 21,7 \\
\hline Cálcio (\%) (Calcium, \%) & 1,02 & 1,02 & 1,02 & 1,02 \\
\hline Fósforo disponível (\%) (Available phosphorus, \%) & 0,46 & 0,46 & 0,46 & 0,46 \\
\hline Lisina total $(\%)$ (Total lysine) & 1,100 & 1,200 & 1,300 & 1,400 \\
\hline Lisina $^{4}(\%)($ Lysine,$\%)$ & 0,995 & 1,090 & 1,201 & 1,293 \\
\hline Metionina $^{4}(\%)$ (Methionine, \%) & 0,393 & 0,482 & 0,554 & 0,643 \\
\hline Met+Cis ${ }^{4}(\%)($ Met + cist,$\%)$ & 0,709 & 0,787 & 0,854 & 0,931 \\
\hline Triptofano $4(\%)$ (Triptofano, \%) & 0,214 & 0,204 & 0,198 & 0,207 \\
\hline Treonina $^{4}(\%)$ (Threonine, \%) & 0,733 & 0,813 & 0,895 & 0,974 \\
\hline Arginina $^{4}(\%)($ L-arginine $)$ & 1,287 & 1,387 & 1,503 & 1,602 \\
\hline Isoleucina $4(\%)$ (L-isoleucine $)$ & 0,869 & 0,922 & 1,030 & 1,122 \\
\hline
\end{tabular}

calculada pela relação entre o ganho de peso $(\mathrm{g})$ e a quantidade de lisina consumida $(\mathrm{g})$.

As análises estatísticas das variáveis estudadas foram realizadas utilizando-se o programa computacional SAEG (Sistema para Análises Estatísticas e Genéticas), desenvolvido na UNIVERSIDADE FEDERAL DE VIÇOSA - UFV (1997), sendo as estimativas das exigências de lisina estabelecidas por meio dos modelos de regressão linear, quadrático e do modelo descontínuo LRP - Linear Response Plateau, conforme o melhor ajuste dos dados.

\section{Resultados e Discussão}

Os valores médios das condições ambientais obtidas durante o período experimental são apresentados na Tabela 2. Com base nos valores de ITGU, pode-se inferir que as aves, no período de 1 a 21 dias, foram submetidas a ambiente de conforto térmico e de alta temperatura.

$\mathrm{Na}$ Tabela 3, são apresentados os resultados de desempenho, consumo de lisina e eficiência de utilização de lisina para ganho, dos pintos de corte 
Tabela 2 - Condições ambientais observadas durante o período experimental Table 2 - Environment conditions observed during the experimental period

\begin{tabular}{|c|c|c|c|c|}
\hline $\begin{array}{l}\text { Idade (dias) } \\
\text { Age (days) }\end{array}$ & $\begin{array}{c}\text { Temperatura do ar }\left({ }^{\circ} \mathrm{C}\right) \\
\text { Air temperature }\end{array}$ & $\begin{array}{c}\text { Umidade relativa }(\%) \\
\text { Relative humity }\end{array}$ & $\begin{array}{c}\text { Temperatura de globo negro }\left({ }^{\circ} \mathrm{C}\right) \\
\text { Black globe temperature }\end{array}$ & ITGU \\
\hline & \multicolumn{4}{|c|}{$\begin{array}{c}\text { Conforto térmico } \\
\text { Thermal comfort }\end{array}$} \\
\hline 1 & $32,1 \pm 0,25$ & $54,5 \pm 1,00$ & $32,3 \pm 0,50$ & $81,6 \pm 0,59$ \\
\hline 2 & $31,5 \pm 0,57$ & $50,8 \pm 2,21$ & $31,5 \pm 0,57$ & $80,8 \pm 0,63$ \\
\hline $3-4$ & $31,0 \pm 0,00$ & $49,7 \pm 3,06$ & $31,1 \pm 0,23$ & $79,8 \pm 0,22$ \\
\hline $5-8$ & $28,8 \pm 0,41$ & $52,5 \pm 2,47$ & $28,9 \pm 0,46$ & $77,1 \pm 0,51$ \\
\hline $9-11$ & $27,9 \pm 0,56$ & $57,4 \pm 2,20$ & $28,0 \pm 0,49$ & $76,3 \pm 0,59$ \\
\hline $12-16$ & $26,4 \pm 0,78$ & $62,6 \pm 3,01$ & $27,1 \pm 0,59$ & $75,2 \pm 0,73$ \\
\hline \multirow[t]{2}{*}{$17-21$} & $24,3 \pm 0,68$ & $65,9 \pm 2,99$ & $25,4 \pm 0,93$ & $72,9 \pm 1,14$ \\
\hline & \multicolumn{4}{|c|}{$\begin{array}{c}\text { Alta temperatura } \\
\text { High temperature }\end{array}$} \\
\hline $1-21$ & $33,2 \pm 3,64$ & $43,2 \% \pm 3,64$ & $33,5 \pm 0,57$ & $82,3 \pm 0,8$ \\
\hline
\end{tabular}

${ }^{1}$ Valores médios (Average values).

alimentados com as rações experimentais, no período de 1 a 21 dias de idade, em diferentes ambientes. Não houve interação entre os níveis de lisina e os ambientes para nenhuma das variáveis estudadas.

As aves mantidas no ambiente de alta temperatura apresentaram redução $(\mathrm{P}<0,05)$ de $3,2 \%$ no ganho de peso (GP) em relação àquelas mantidas no conforto térmico. Resultados similares foram obtidos por GERAERT et al. (1996) e BAZIZ et al. (1996).

$\mathrm{O}$ ganho de peso das aves criadas sob condições de conforto térmico foi influenciado $(\mathrm{P}<0,09)$ pelos níveis de lisina da ração de forma linear crescente, até o nível de 1,4\% de lisina total (Tabela 4). Este nível de lisina se encontra acima daquele de $1,10 \%$ de lisina total recomendado pelo NATIONAL RESEARCH COUNCIL - NRC (1994) e de 1,16\% obtido por ROSTAGNO et al. (1996), contudo ficou próximo ao valor de $1,34 \%$ obtido por VALERIO et al. (1999a) e de 1,39\% estimado por SURISDIARTO e FARRELL (1991), quando usaram rações com mínimo excesso de aminoácidos.

O resultado obtido neste trabalho demonstrou que o nível de lisina recomendado pelo NRC (1994) e por ROSTAGNO et al. (1996) pode estar subestimando as exigências para GP de frangos de corte de 1 a 21 dias. Portanto, ao se avaliarem níveis de lisina para frangos de corte, a manutenção da relação da lisina com os demais aminoácidos é essencial para possibilitar ao animal expressar todo o seu potencial genético. Segundo KIDD et al. (1997), aumentar o nível de lisina na ração, sem considerar os demais aminoácidos, pode resultar em desempenho limitado por deficiência de algum outro aminoácido essencial.
Com relação às aves mantidas em alta temperatura, observou-se efeito $(\mathrm{P}<0,07)$ dos níveis de lisina sobre o GP, que também aumentou de forma linear. No entanto, o modelo descontínuo "Linear Response Plateu" - LRP foi o que melhor que se ajustou aos dados, estimando-se em $1,24 \%$ o nível de lisina total, a partir do qual ocorreu um platô (Tabela 4). De forma semelhante, VALERIO et al. (1999b), avaliando níveis de lisina ( 1,16 a 1,34\%) para frangos de corte, de 1 a 21 dias de idade, mantidos em ambiente com temperatura de $33^{\circ} \mathrm{C}$, constataram aumento linear no GP das aves.

Verificou-se efeito $(\mathrm{P}<0,01)$ da temperatura ambiente sobre o consumo de ração (CR), com as aves no ambiente de alta temperatura consumindo $11,5 \%$ menos. Resultado semelhante foi observado por LEESON E SUMMERS (1991), HAHN E BAKER (1993) e ROSTAGNO (1995), que também constataram redução no $\mathrm{CR}$ das aves, devido ao aumento na temperatura ambiente. A redução ocorrida no CR justifica a diminuição verificada no GP das aves sob estresse de calor. Os níveis de lisina não influenciaram $(\mathrm{P}>0,10)$ o consumo de ração das aves em ambos os ambientes. De acordo com Parr e Summers (1991), citados por VALERIO et al. (1999b), além da energia, o imbalanço entre os aminoácidos da ração é um fatores que influenciam a ingestão de alimento das aves. Dessa forma, pode-se inferir que o fato de se manter a relação entre a lisina e os aminoácidos mais críticos para as aves (metionina, treonina, isoleucina, valina e triptofano) nos diferentes níveis de lisina avaliados, além das rações terem sido isoenergéticas e isoproteicas, justifica os consu- 
Rev. bras. zootec.

Tabela 3 - Desempenho, consumo de lisina e eficiência de utilização de lisina para ganho de pintos de corte de 1 a 21 dias de idade, em diferentes ambientes térmicos

Table 3 - Performance, lysine intake and efficiency of the use of lysine for gain for broilers from 1 to 21 -old in different thermal environments

\begin{tabular}{|c|c|c|c|c|c|c|}
\hline & \multicolumn{4}{|c|}{$\begin{array}{c}\text { Nível de lisina total }(\%) \\
\text { Total lysine level }\end{array}$} & \multirow[t]{2}{*}{$\begin{array}{l}\text { Média }{ }^{1} \\
\text { Mean }\end{array}$} & \multirow[t]{2}{*}{$\mathrm{CV}$} \\
\hline & 1,1 & 1,2 & 1,3 & 1,4 & & \\
\hline \multicolumn{7}{|l|}{ Conforto térmico } \\
\hline Ganho de peso médio $(\mathrm{g})^{2}$ & 636 & 655 & 660 & 673 & $657^{\mathrm{A}}$ & 4,48 \\
\hline $\begin{array}{l}\text { Average daily gain } \\
\text { Consumo de ração (g) }\end{array}$ & 908 & 940 & 944 & 948 & $937^{\mathrm{A}}$ & 4,43 \\
\hline Feedintake & & & & & & \\
\hline $\begin{array}{l}\text { Conversão alimentar (g) } \\
\text { Feed:gain ratio }\end{array}$ & 1,44 & 1,43 & 1,43 & 1,42 & $1,43^{\mathrm{A}}$ & 3,82 \\
\hline $\begin{array}{l}\text { Consumo de lisina }(\mathrm{g})^{4} \\
\text { Lysine intake }\end{array}$ & 10,0 & 11,3 & 12,3 & 13,3 & $11,7^{\mathrm{A}}$ & 4,51 \\
\hline $\begin{array}{l}\text { Efic. utilização lisina/ganho }(\mathrm{g} / \mathrm{g})^{3} \\
\text { Effic. lysine/gain }\end{array}$ & 63,6 & 58,0 & 53,7 & 50,6 & $56,4^{\mathrm{A}}$ & 3,68 \\
\hline \multicolumn{7}{|c|}{$\begin{array}{l}\text { Alta temperatura } \\
\text { High temperature }\end{array}$} \\
\hline $\begin{array}{l}\text { Ganho de peso médio }(\mathrm{g})^{2} \\
\text { Average daily gain }\end{array}$ & 617 & 637 & 648 & 641 & $636^{\mathrm{B}}$ & 3,30 \\
\hline $\begin{array}{l}\text { Consumo de ração }(\mathrm{g}) \\
\text { Feed intake }\end{array}$ & 832 & 828 & 818 & 836 & $829^{\mathrm{B}}$ & 3,69 \\
\hline $\begin{array}{l}\text { Conversão alimentar }(\mathrm{g})^{5} \\
\text { Feed:gain ratio }\end{array}$ & 1,34 & 1,30 & 1,25 & 1,30 & $1,30^{\mathrm{B}}$ & 3,25 \\
\hline $\begin{array}{l}\text { Consumo de lisina }(\mathrm{g})^{3} \\
\text { Lysine intake }\end{array}$ & 9,2 & 9,9 & 10,6 & 11,7 & $10,3^{\mathrm{B}}$ & 3,29 \\
\hline $\begin{array}{l}\text { Efic. lisina/ganho }(\mathrm{g})^{3} \\
\text { Effic. Lysine/gain }\end{array}$ & 67,0 & 64,3 & 61,1 & 54,8 & $62,1^{\mathrm{B}}$ & 3,09 \\
\hline $\begin{array}{ll}1 & \text { Médias seguidas de letras diferen } \\
& \text { by } F \text { test }[P<05]) . \\
2,3 \text { e } 4 & \text { Efeito linear }(P<0,09), \quad(P<0,07) \text { ( }) \text { Efeito quadrático }(P<0,02)(\text { Quad } \\
5 & \text { Efoil }\end{array}$ & na $n$ & nac & teste & & $\begin{array}{l}\text { fferent lette } \\
\text { respectivel }\end{array}$ & \\
\hline
\end{tabular}

mos semelhantes observados entre os tratamentos nos diferentes ambientes.

A conversão alimentar (CA) também foi influenciada $(\mathrm{P}<0,05)$ pela temperatura ambiente, sendo que os menores valores de CA foram observados nas aves mantidas no ambiente de calor. Resultados semelhantes foram obtidos por BAZIZ et al. (1990) e ZANUSSO (1998), que observaram melhora na CA de frangos de corte de 1 a 21 dias de idade mantidos sob condições de estresse por calor.

Em contrapartida, HAN e BAKER (1993) verificaram piora na eficiência alimentar de frangos de corte de 8 a 21 dias de idade, expostos a altas temperaturas em relação àqueles mantidos em ambiente de conforto térmico.

Com relação aos resultados obtidos em cada ambiente, constatou-se que, enquanto no conforto a CA não foi influenciada $(\mathrm{P}>0,10)$, pelos níveis de lisina, no de calor, ocorreu efeito $(\mathrm{P}<0,02)$ quadrático, com a CA melhorando até o nível de 1,285\%, correspondente a um consumo estimado de $10,4 \mathrm{~g}$ de lisina total no período (Tabela 4). Embora o resultado do nível de lisina que proporcionou o melhor valor de $\mathrm{CA}$ neste trabalho tenha ficado abaixo daquele de 1,34\% determinado por VALERIO et al. (1999b), para frangos de corte de 1 a 21 dias de idade mantidos em condição de calor, em termos de consumo de lisina, os resultados foram similares, correspondendo a 10,4 e $10,8 \mathrm{~g}$, respectivamente.

As aves mantidas sob condições de conforto térmico apresentaram maior $(\mathrm{P}<0,05)$ consumo de lisina no período de 1 a 21 dias, correspondendo a $13,6 \%$, em relação àquelas mantidas no ambiente de alta temperatura, o que ocorreu em razão da maior ingestão de alimentos observada para as aves criadas no ambiente de conforto térmico.

O consumo de lisina aumentou $(\mathrm{P}<0,01)$ de forma linear, em razão dos crescentes níveis de lisina da 
Tabela 4 - Regressão dos efeitos de lisina total sobre ganho de peso médio e conversão alimentar de pintos de corte mantidos em ambiente de conforto térmico e alta temperatura

Table 4 - Regression of the total lysine levels on average weight gain and feed:gain ratio of broilers under thermal comfort and high temperature environments

\begin{tabular}{|c|c|c|}
\hline $\begin{array}{l}\text { Variável } \\
\text { Variable }\end{array}$ & $\begin{array}{l}\text { Regressão } \\
\text { Regression }\end{array}$ & \\
\hline & $\begin{array}{l}\text { Conforto térmico } \\
\text { Thermal comfort }\end{array}$ & \\
\hline \multirow{3}{*}{$\begin{array}{l}\text { Ganho de peso } \\
\text { Weight gain }\end{array}$} & Linear $-\hat{\mathrm{Y}}=516,494+111,728 \mathrm{Lis}$ & $\mathrm{R} 2=0,94$ \\
\hline & & \\
\hline & $\begin{array}{l}\text { Alta temperatura } \\
\text { High temperature }\end{array}$ & \\
\hline Ganho de peso médio & Linear $-\hat{\mathrm{Y}}=529,882+84,868 \mathrm{Lis}$ & $\mathrm{R} 2=0,64$ \\
\hline Average weight gain & LRP $-\hat{Y}=436,664+165,00 \mathrm{Lis}$ & $\mathrm{R} 2=0,98$ \\
\hline $\begin{array}{l}\text { Conversão alimentar } \\
\text { Feed:gain ratio }\end{array}$ & Quadrático - $\hat{\mathrm{Y}}=5,142-6,029 \mathrm{Lis}+2,3449 \mathrm{Lis}^{2}$ & $\mathrm{R} 2=0,90$ \\
\hline
\end{tabular}

ração, em ambos os ambientes térmicos. O aumento do consumo de lisina, independente do ambiente, influenciou positivamente o ganho de peso da aves. Da mesma forma, CONHALATO (1998), trabalhando com pintos de corte na fase inicial, observou aumento no ganho de peso das aves, à medida que o consumo de lisina se elevou. Embora os níveis de lisina tenham piorado $(\mathrm{P}<0,01)$ a eficiência de utilização de lisina para GP, em ambos os ambientes, as aves submetidas à alta temperatura apresentaram melhores resultados $(\mathrm{P}<0,01)$ em relação àquelas mantidas no conforto térmico. Considerando os resultados obtidos por LEE et al. (1996), com suínos em crescimento, em que a eficiência de utilização de lisina (ganho de peso:lisina consumida) foi reduzida, quando a procentagem de lisina em relação à $\mathrm{PB}$ da ração aumentou de 5,2 para $6,7 \%$, pode-se deduzir que, entre outros fatores, a elevação da relação lisina:proteína de 5,0 até 6,5\% ocorrida entre os tratamentos, neste trabalho, pode ter contribuído para a redução ocorrida na eficiência de utilização de lisina para ganho de peso das aves nos dois ambientes avaliados. Já no ambiente de calor, a possível redução do peso relativo dos órgãos e das vísceras, principalmente, fígado e intestino delgado, que reconhecidamente apresentam alto turnover de proteína, pode ter contribuído para a maior eficiência de utilização de lisina para ganho e redução da exigência de lisina.

Avaliando níveis de lisina para frangos de corte de 1 a 21 dias, submetidos a diferentes temperaturas ambientais, VALERIO et al. (1999b) verificaram redução no peso relativo de vísceras e intestino delgado, das aves mantidas sob estresse por calor.

Os frangos mantidos em ambiente de alta temperatura, além de apresentarem maior eficiência média de utilização de lisina para ganho, tiveram menor redução (18,2\%) desta eficiência entre os níveis de lisina, quando comparados àqueles em ambiente de conforto $(20,4 \%)$.

$\mathrm{Na}$ alta temperatura, a redução acentuada na eficiência de utilização de lisina para ganho de 11,5\% verificada entre os níveis de 1,3 e 1,4\% de lisina estaria indicando que a máxima deposição de proteína pode ter ocorrido no nível de $1,3 \%$ de lisina, o que não foi constatado no conforto.

\section{Conclusões}

A alta temperatura ambiente influenciou negativamente o ganho de peso e o consumo de ração, embora tenha melhorado a conversão alimentar das aves em relação ao ambiente de conforto térmico.

Frangos de corte de 1 a 21 dias exigem 1,4\% de lisina total, quando mantidos em ambiente termoneutro, e $1,285 \%$, quando expostos à alta temperatura.

\section{Referências Bibliográficas}

BAKER, D.H., HAN, Y. 1994. Ideal amino acid profile for chicks during the first three weeks posthatching. Poult. Sci., 73:1441-1447.

BARBOZA, W.A. Exigência nutricional de lisina para duas marcas comerciais de frango de corte. Viçosa: UFV, 1998. 115 p. Tese (Doutorado em Zootecnia) - Universidade Federal de Viçosa, 1998. 
Rev. bras. zootec.

BAZIZ, H.A., GERAERT, P.A., GUILLAUMIN, S. Effects of high temperature and dietary composition on growth, body and composition energy retention in broilers. In: EUROPEAN POULTRY CONFERENCE, 7, BARCELONA, 1990. Proceedings... Barcelona, 1990. p.626-629.

BAZIZ, H.A., GERAERT, P.A., GUILLAUMIN, S. 1996. Chronic heat exposure enhances fat deposition and modifies muscle and far partition in broiler carcasses. Poult. Sci., 75:505-513.

BUFFINGTON, D.E., COLAZZO-AROCHO, A., CANTON, G.H. et al. 1977. Black globe-umidity confort index for dairy cows. St. Joseph, Michigan: Amer. Soc. Agric. Eng. 19p.

CONHALATO, G.S. Exigência de lisina digestível parafrangos de corte machos. Viçosa: UFV, 1998. 68p. Dissertação (Mestrado em Zootecnia) - Universidade Federal de Viçosa, 1998.

CURTIS, S.E. 1983. Environmental management in animal agriculture. Ames, Iowa: Iowa State University Press. 409p.

GERAERT, P.A., PADILHA, J.C.F., GUILLAUMIN, S. 1996. Metabolic and endocrine changes induced by chronic heat exposure in broiler chickens: growth performance, body composition and energy retention. Br. Poult. Nut., 75(2):1955-204.

HAN, Y., BAKER, D.H. 1993. Effects of sex, heat stress, body weight, and genetic strain on the dietary lysine requirement of broiler chicks. Poult. Sci., 72(4):701-708.

KIDD, M.T., KERR, B.J. ANTHONY, N.B. 1997. Dietary interactions between lysine and threonine in broilers. Poult. Sci., 76:608-614.

LEESON, S., SUMMERS, J.D. 1991. Commercial poultry nutrition. Guelp, Ontario: University Books. 335p.

LEE, K.U., BOYD, D., AUSTIC, R.E. 1996. Metabolic efficiency of dietary protein and lysine utilization by growing pigs. (file:///C1/2/AAANPPC/96LEE- 1.HTM).

NATIONAL RESEARCH COUNCIL - NRC. 1994. Nutrient requirement of poultry. 9.ed. Washington, D.C.: National Academy Press. 155p.

PACK, M. 1995. Proteína ideal para frangos de corte. Conceitos e posição atual. In: CONFERÊNCIA APINCO DE CIÊNCIA E TECNOLOGIAS AVÍCOLAS, p. 95-110.

PENZ, A.M. 1989. Estresse pelo calor: Efeitos em frangos de corte e matrizes. Manipulação do equilíbrio ácido-base. In: CONFERÊNCIA APINCO 1989 DE CIÊNCIA E TECNOLOGIA AVÍCOLA, São Paulo. 1989. p.139-146.

RHODIMET feed formulation guide. 1993. 6.ed. France: RhônePoulenc Animal Nutrition. 39p.

ROSTAGNO, H.S. Programas de alimentação e nutrição para frangos de corte adequados ao clima. In: SIMPÓSIO INTERNACIONAL SOBRE AMBIÊNCIA E INSTALAÇÃO NA AVICULTURA INDUSTRIAL, 1995, Campinas, SP. Anais... Campinas, 1995. p.11-20.
ROSTAGNO, H.S., BARBARINO JR., P., BARBOZA, W.A. Exigências nutricionais das aves determinadas no Brasil. In: SIMPÓSIO INTERNACIONAL SOBRE EXIGÊNCIAS NUTRICIONAIS DE AVES E SUÍNOS, 1996, Viçosa. Anais... Viçosa, 1996. p.361.

SURISDIARTO, A., FARRELL, D.J. 1991. The relationship between dietary crude protein and dietary lysine requirement by broiler chicks on diets with and without the 'ideal' amino acids balance. Poult. Sci., 70:830-836.

UNIVERSIDADE FEDERAL DE VIÇOSA - UFV. 1997. SAEG - Sistemas de análises estáticas e genéticas. Versão 7.1. Viçosa, MG. 150p. (Manual do usuário).

VALERIO, S.R., OLIVEIRA, R.F.M., DONZELE, J.L. et al. Níveis de lisina digestível mantendo a relação aminoacídica para frangos de corte de 1 a 21 dias de idade mantidos em ambiente de conforto térmico. REUNIÃO ANUAL DA SOCIEDADE BRASILEIRA DE ZOOTECNIA, 36, 1999, Porto Alegre. Anais... Porto Alegre: SBZ, 1999a. p.195

VALERIO, S.R., OLIVEIRA, R.F.M., DONZELE, J.L. et al. Níveis de lisina digestível mantendo a relação aminoacídica para frangos de corte de 1 a 21 dias de idade, mantidos em ambiente de estresse térmico. REUNIÃO ANUAL DA SOCIEDADE BRASILEIRA DE ZOOTECNIA, 36, 1999, Porto Alegre. Anais... Porto Alegre: SBZ, 1999b. p.196

ZANUSSO, J.T. Níveis de energia metabolizável para frangos de corte de 1 a 21 dias de idade mantidos em ambiente de conforto e estresse térmico. Viçosa: UFV, 1998. 64p. Dissertação (Mestrado em Zootecnia) - Universidade Federal de Viçosa, 1998.

Recebido em: 24/03/00 Aceito em: 09/11/00 